Network Working Group

Request for Comments: 2456

Category: Standards Track
B. Clouston Cisco Systems

B. Moore

IBM Corporation

November 1998

\title{
Definitions of Managed Objects \\ for APPN TRAPS
}

\section{Status of this Memo}

This document specifies an Internet standards track protocol for the Internet community, and requests discussion and suggestions for improvements. Please refer to the current edition of the "Internet Official Protocol Standards" (STD 1) for the standardization state and status of this protocol. Distribution of this memo is unlimited.

Copyright Notice

Copyright (C) The Internet Society (1998). All Rights Reserved.

Abstract

This memo defines a portion of the Management Information Base (MIB) for use with network management protocols in the Internet community. In particular, it defines objects for receiving notifications from network devices with APPN (Advanced Peer-to-Peer Network) and DLUR (Dependent LU Requester) capabilities. This memo identifies notifications for the APPN and DLUR architecture.

Table of Contents

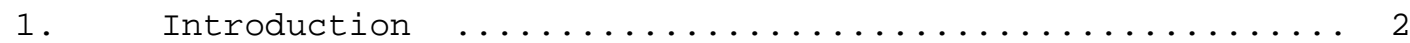

2. The SNMP Network Management Framework ............ 2

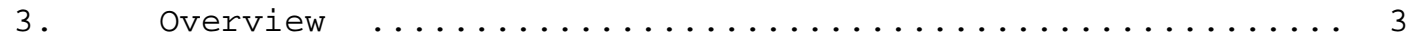

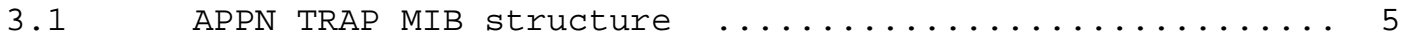

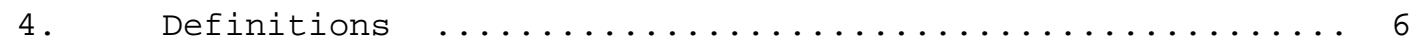

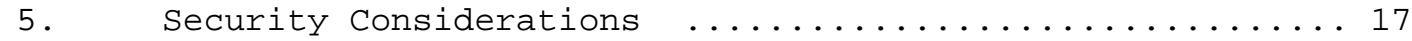

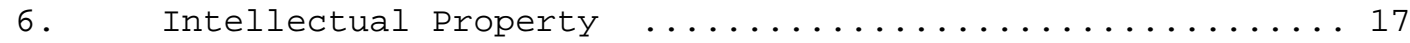

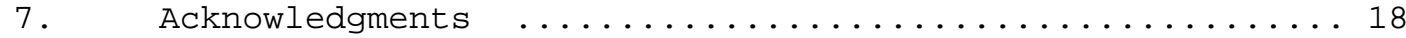

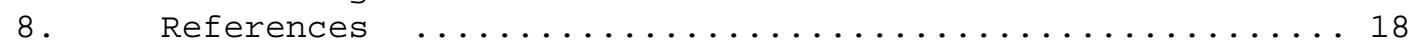

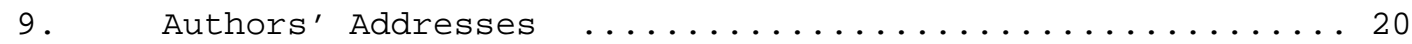

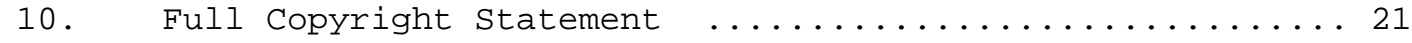




\section{Introduction}

This document is a product of the SNA NAU Services MIB Working Group. It defines a MIB module for notifications for devices with Advanced Peer-to-Peer Networking (APPN) and Dependent LU Requester (DLUR) capabilities.

The key words "MUST", "MUST NOT", "REQUIRED", "SHALL", "SHALL NOT", "SHOULD", "SHOULD NOT", "RECOMMENDED", "MAY", and "OPTIONAL" in this document are to be interpreted as described in RFC 2119 [13].

2. The SNMP Network Management Framework

The SNMP Management Framework presently consists of five major components:

o An overall architecture, described in RFC 2271 [1] .

- Mechanisms for describing and naming objects and events for the purpose of management. The first version of this structure of Management Information (SMI) is called SMIv1 and described in $\mathrm{STD}$ 16, RFC 1155 [2], STD 16, RFC 1212 [3] and RFC 1215 [4]. The second version, called SMIv2, is described in RFC 1902 [5], RFC 1903 [6] and RFC 1904 [7].

- Message protocols for transferring management information. The first version of the SNMP message protocol is called SNMPV1 and described in STD 15, RFC 1157 [8]. A second version of the SNMP message protocol, which is not an Internet standards track protocol, is called SNMPv2c and described in RFC 1901 [9] and RFC 1906 [10]. The third version of the message protocol is called SNMPv3 and described in RFC 1906 [10], RFC 2272 [11] and RFC 2274 [12].

- Protocol operations for accessing management information. The first set of protocol operations and associated PDU formats is described in STD 15, RFC 1157 [8]. A second set of protocol operations and associated PDU formats is described in RFC 1905 [13].

- A set of fundamental applications described in RFC 2273 [14] and the view-based access control mechanism described in RFC 2275 [15].

Managed objects are accessed via a virtual information store, termed the Management Information Base or MIB. Objects in the MIB are defined using the mechanisms defined in the SMI. 
This memo specifies a MIB module that is compliant to the SMIv2. A MIB conforming to the SMIv1 can be produced through the appropriate translations. The resulting translated MIB must be semantically equivalent, except where objects or events are omitted because no translation is possible (use of Counter64). Some machine readable information in SMIV2 will be converted into textual descriptions in SMIv1 during the translation process. However, this loss of machine readable information is not considered to change the semantics of the MIB.

\section{Overview}

This document identifies the set of objects for reporting the status of devices with APPN and DLUR capabilities via notifications.

See the SNANAU APPN MIB [18] and SNANAU DLUR MIB [19] for the objects for monitoring the configuration and active characteristics of the devices with APPN and DLUR capabilities. Many objects contained in the notifications of this MIB are imported from the APPN and DLUR MIBs. Implementors of this MIB must also implement the APPN MIB. Implementations that support the appnTrapMibDlurConfGroup and the appnTrapMibDlurNotifGroup must also implement the DLUR MIB.

The SNANAU APPN MIB allows a management station to collect the network topology of an APPN network (the network nodes (NNs) in the network and all of transmission groups (TGs) between the network nodes) from an APPN device. It also allows the management station to collect the local topology (TGs to end stations, and locally defined ports and link stations) from an APPN device. While the SNANAU APPN MIB has an efficient way to poll the APPN device for updates to the network topology, using flow reduction sequence numbers (FRSNs) as a table index; it does not have a mechanism to poll the local topology tables (appnLocalTgTable, appnportTable, and appnLsTable) for status changes.

This MIB provides a mechanism for an APPN device to send notifications to inform the management station of status changes to rows of these tables. Status changes include operational state changes, and for TGs also include control-point to control-point $(\mathrm{CP}-\mathrm{CP})$ session state changes. A notification is defined for each type of status change for each table.

The port and link operational state objects have intermediate states. Notifications are only sent for transition to active or inactive state. 
Notifications are only sent for row creation if the state is active or operational. This is done to avoid sending a notification as the row is created with an inactive initial state, followed by another notification as the resource is activated.

Notifications are only sent for row deletion if the last state was active or operational. In most cases, a resource must be deactivated before it can be deleted, and the deactivation will cause a notification to be sent. There is no need for a second notification to be sent for the row deletion, except for the case where the deletion occurred without deactivation. In this case, the state of the object in the notification will indicate an inactve state, since a deleted resource can no longer be active.

The purpose of the appnLocalTgCpCpstatechangeTrap notification is to identify the loss or recovery of $\mathrm{CP}-\mathrm{CP}$ sessions on a $\mathrm{TG}$ while the $\mathrm{TG}$ remains operational. Thus this notification is only sent if there is a change to an appnLocalTgCpCpsession object, but not a change to the appnLocalTgoperational object. This notification is never sent for the creation or deletion of a row in the appnLocalTgTable.

Each notification always contains an object which is a count of the number of times the status of a row in table has changed since the APPN node was last reinitialized. This enables a management station to detect that it has missed a notification, if it does not get the notifications in numerical sequence. If the notifications are not in sequence, the management station should retrieve the entire table to get the correct status for all rows.

Similarly, the SNANAU DLUR MIB provides a mechanism for retrieving the configuration and status of dependent LU server (DLUS) sessions on a device with DLUR capabilities. This MIB defines a notification for a DLUR-DLUS session state change of a row in the dlurDlustable, in the manner described above. A notification is only sent for a session state transition to active or inactive. As with the above notifications, it is only sent on row creation if the initial state is active; and on row deletion is the last state was active, in which case the notification indicates that the state is now inactive.

The SNANAU APPN MIB also provides a mechanism for a management station to collect traffic statistics on intermediate sessions, primarily for accounting purposes. However, when the session is terminated, all statistics from the last poll until the session termination time are lost, since the row for that session is deleted from the appnisinTable. This MIB defines a notification so that the session's final statistics can be sent to a management station. If the notification is not delivered, the final session statistics are lost. If this is a concern, polling of the appnIsInTable in the APPN 
MIB should be increased to more likely reduce the time between the last poll and the session termination, thereby reducing the amount of data lost.

Highlights of the management functions supported by the APPN TRAP MIB module include the following:

- A notification for an APPN local TG operational state change.

- A notification for an APPN local TG CP-CP session state change.

- A notification for an APPN port operational state change.

- A notification for an APPN link station operational state change.

- A notification for a DLUR-DLUS session state change.

- A notification for reporting final APPN intermediate session statistics.

This MIB module does not support:

o Objects to query the configuration or status of APPN nodes on demand.

o Notifications for changes to local topology tables not related to status.

\subsection{APPN TRAP MIB structure}

The APPN TRAP MIB module contains a group of notifications, and a group of supporting objects.

The group of notifications consists of the following notifications:

1) appnIsrAccountingDataTrap

This notification is generated by an APPN device when an intermediate session is terminating, to report the final accounting statistics of the session.

2) appnLocalTgoperStateChangeTrap

This notification identifies a change to the appnLocalTgoperational object in a row of the SNANAU APPN MIB appnLocalTgTable. 


\section{3) appnLocalTgCpCpStateChangeTrap}

This notification identifies a change to the appnLocalTgCpCpSession object in a row of the SNANAU APPN MIB appnLocalTgTable.

4) appnPortoperStateChangeTrap

This notification identifies a change to the appnPortoperstate object in a row of the SNANAU APPN MIB appnPortTable.

5) appnLsOperStateChangeTrap

This notification identifies a change to the appnLsoperstate object in a row of the SNANAU APPN MIB appnLsTable.

6) dlurDlusstateChangeTrap

This notification identifies a change to the dlurDlussessnstatus object in a row of the SNANAU DLUR MIB dlurDlusTable.

The group of supporting objects contains the appnTrapControl object, which controls whether the APPN device generates each type of notification. Note that generation of the appnIsrAccountingDataTrap is not controlled by this object; instead it is controlled by the appnIsInGlobalCtrAdminStatus object in the SNANAU APPN MIB.

Although APPN notification generation could be controlled solely by entries in the snmpNotificationMIB, RFC 2273 [9], the appnTrapControl object exists in this MIB so that implementations are not required to implement RFC 2273 to control generation of APPN notifications. For a notification to be generated and sent as a TRAP or INFORM, the notification type must first be enabled by the appnTrapcontrol object. It must also not be disabled by an snmpNotificationMIB entry. The destination of notifications is not within the scope of this MIB.

Also contained in this group are objects for the TG, port, link, and DLUR-DLUS session notifications to indicate the number of times each of the tables has had a status change of a row since the APPN node was last reinitialized.

4. Definitions

APPN-TRAP-MIB DEFINITIONS $::=$ BEGIN

IMPORTS

Counter32, OBJECT-TYPE, MODULE-IDENTITY, 


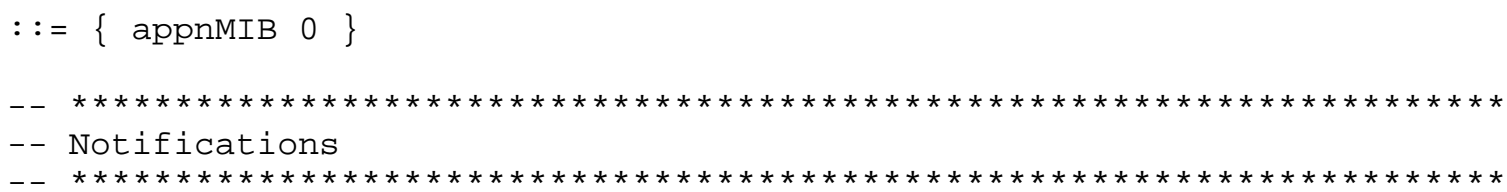

"When it has been enabled, this notification is generated by an APPN node whenever an ISR session passing through the node is taken down, regardless of whether the session went down normally or abnormally. Its purpose is to allow a management application (primarily an accounting application) that is monitoring the ISR counts to receive the final values of these counts, so that the application can properly account for the amounts the counts were incremented since the last time the application polled them. The appnIsInSessUpTime object provides the total amount of time that the session was active.

This notification is not a substitute for polling the ISR counts. In particular, the count values reported in this notification cannot be assumed to be the complete totals for the life of the session, since they may have wrapped while the session was up.

The session to which the objects in this notification apply is identified by the fully qualified CP name and PCID that make up the table index. An instance of this notification will contain exactly one instance of each of its objects, and these objects will all belong to the same conceptual row of the appnIsInTable.

Generation of this notification is controlled by the same object in the APPN MIB, appnIsInGlobeCtrAdminstatus, that controls whether the count objects themselves are being incremented." 
$::=\{$ appnTrapMiB 1$\}$

appnLocalTgOperStateChangeTrap NOTIFICATION-TYPE OBJECTS \{

appnLocalTgTablechanges, appnLocalTgoperational \}

STATUS current DESCRIPTION

"When it has been enabled, this notification makes it possible for an APPN topology application to get asynchronous notifications of local TG operational state changes, and thus to reduce the frequency with which it polls for these changes.

This notification is sent whenever there is a change to the appnLocalTgoperational object in a row of the appnLocalTgTable. This notification is only sent for row creation if the row is created with a value of 'true' for appnLocalTgoperational. This notification is only sent for row deletion if the last value of appnLocalTgoperational was 'true'. In this case, the value of appnLocalTgoperational in the notification shall be 'false', since the deletion of a row indicates that the $\mathrm{TG}$ is no longer operational.

The notification is more than a simple 'poll me now' indication. It carries both a count of local TG topology changes, and the current operational state itself. The count of changes allows an application to detect lost notifications, either when polling or upon receiving a subsequent notification, at which point it knows it must retrieve the entire appnLocalTgTable again. This is the same count as used in the appnLocalcpcpstatechangeTrap. A lost notification could indicate a local TG CP-CP session state change or an operational state change.

Generation of this notification is controlled by the appnTrapControl object."

$::=\{$ appnTrapMiB 2$\}$

appnLocalTgCpCpChangeTrap NOTIFICATION-TYPE OBJECTS \{ appnLocalTgTablechanges, \} appnLocalTgCpCpSession

STATUS current DESCRIPTION

"When it has been enabled, this notification makes it possible 
for an APPN topology application to get asynchronous

notifications of local TG control-point to control-point (CP-CP) session state changes, and thus to reduce the

frequency with which it polls for these changes.

This notification is sent whenever there is a change to the appnLocalTgCpCpSession object but NOT the appnLocalTgoperational object in a row of the appnLocalTgTable. This notification is never sent for appnLocalTgTable row creation or deletion.

The notification is more than a simple 'poll me now' indication. It carries both a count of local TG topology changes, and the current $\mathrm{CP}-\mathrm{CP}$ session state itself. The count of changes allows an application to detect lost notifications, either when polling or upon receiving a subsequent notification, at which point it knows it must retrieve the entire appnLocalTgTable again. This is the same count as used in the appnLocalTgOperstateChangeTrap. A lost notification could indicate a local TG CP-CP session state change or an operational state change.

Generation of this notification is controlled by the appnTrapcontrol object."

$::=\{$ appnTrapMiB 3$\}$

appnPortOperStateChangeTrap NOTIFICATION-TYPE

OBJECTS \{

appnPortTablechanges,

appnPortoperstate \}

STATUS current

DESCRIPTION

"When it has been enabled, this notification makes it possible for an APPN topology application to get asynchronous notifications of port operational state changes, and thus to reduce the frequency with which it polls for these changes. This notification is only sent when a appnPortoperstate has transitioned to a value of 'active' or 'inactive'.

This notification is sent whenever there is a appnPortoperstate object transition to 'inactive' or 'active' state in the appnportTable. This notification is only sent for row creation if the row is created with a value of 'active' for appnPortoperstate. This notification is only sent for row deletion if the last value of appnPortoperstate was 'active'. In this case, the value of appnportoperstate 
in the notification shall be 'inactive', since the deletion of a row indicates that the port is no longer active.

The notification is more than a simple 'poll me now' indication. It carries both a count of port table changes, and the operational state itself. The count of changes allows an application to detect lost notifications, either when polling or upon receiving a subsequent notification, at which point it knows it must retrieve the entire appnPortTable again.

Generation of this notification is controlled by the appntrapcontrol object."

$::=\{$ appnTrapMiB 4$\}$

appnLsOperStateChangeTrap NOTIFICATION-TYPE OBJECTS \{ appnLsTablechanges, \}

STATUS current DESCRIPTION

"When it has been enabled, this notification makes it possible for an APPN topology application to get asynchronous notifications of link station operational state changes, and thus to reduce the frequency with which it polls for these changes. This notification is only sent when a appnLsoperstate has transitioned to a value of 'active' or 'inactive'.

This notification is sent whenever there is a appnLsoperstate object transition to 'inactive' or 'active' state in the appnLstable. This notification is only sent for row creation if the row is created with a value of 'active' for appnLsoperstate. This notification is only sent for row deletion if the last value of appnLsoperstate was 'active'. In this case, the value of appnLsoperstate in the notification shall be 'inactive', since the deletion of a row indicates that the link station is no longer active.

The notification is more than a simple 'poll me now' indication. It carries both a count of link station table changes, and the operational state itself. The count of changes allows an application to detect lost notifications, either when polling or upon receiving a subsequent notification, at which point it knows it must retrieve the entire appnLsTable again.

Generation of this notification is controlled by the appnTrapControl object." 


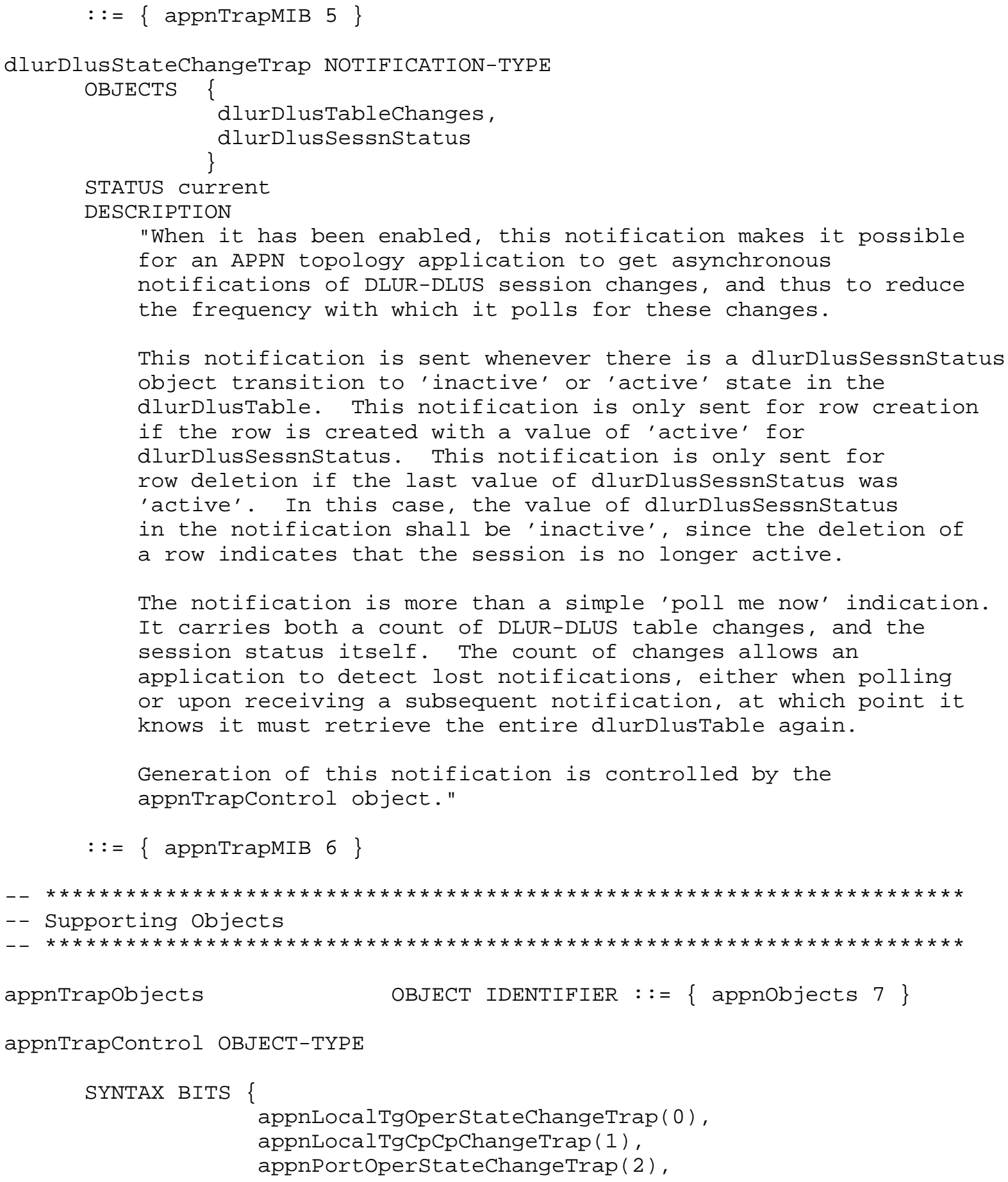




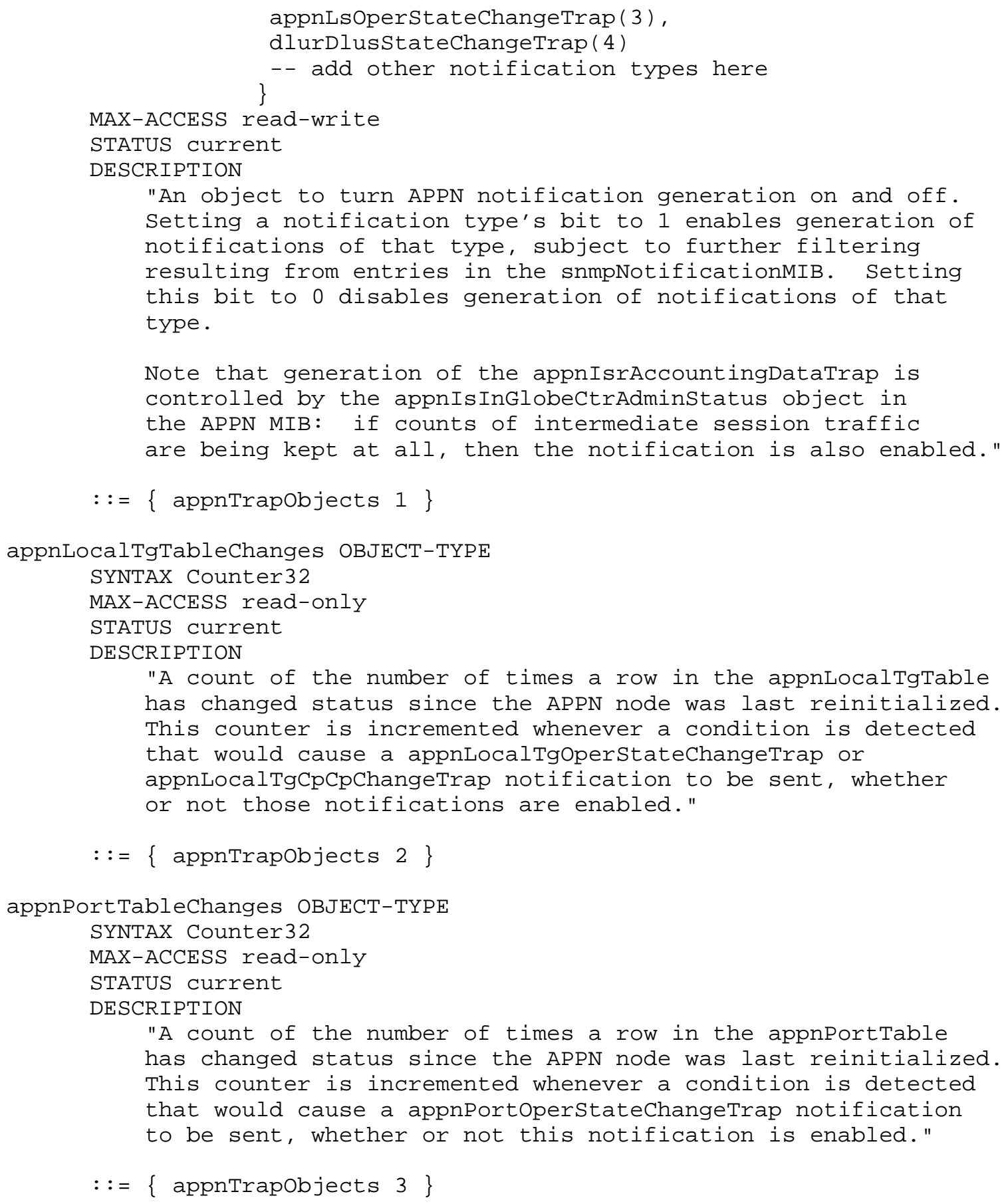




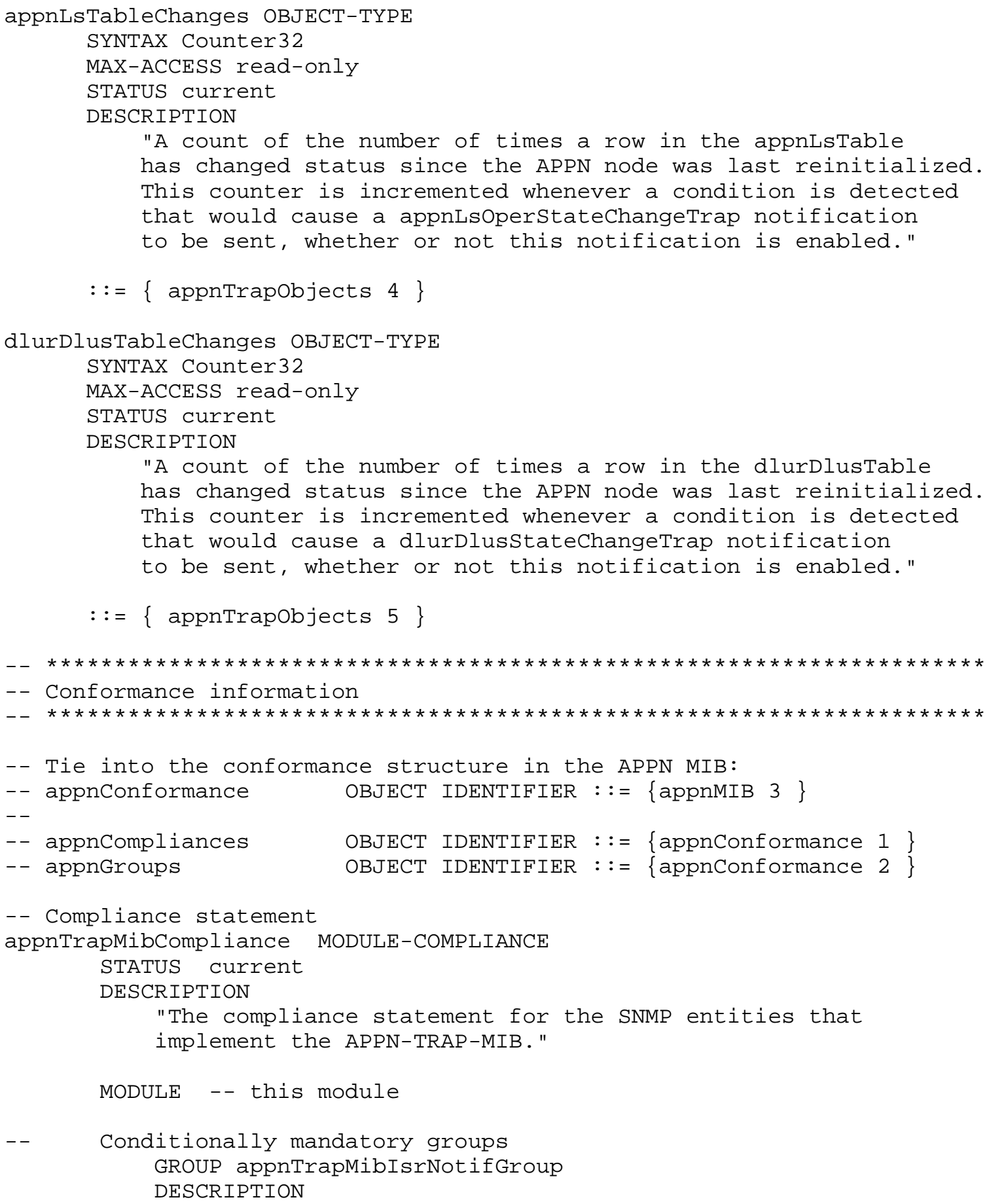




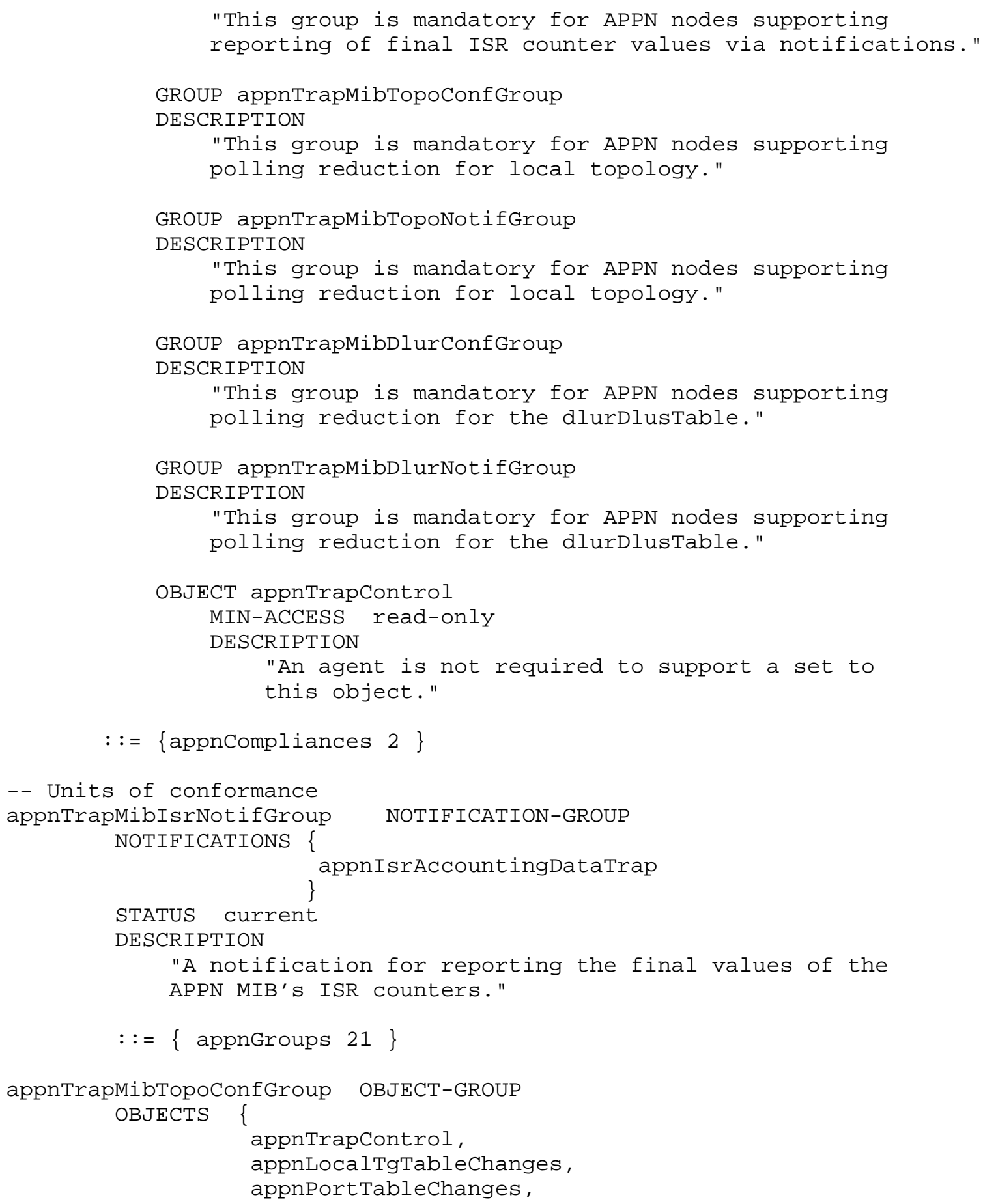




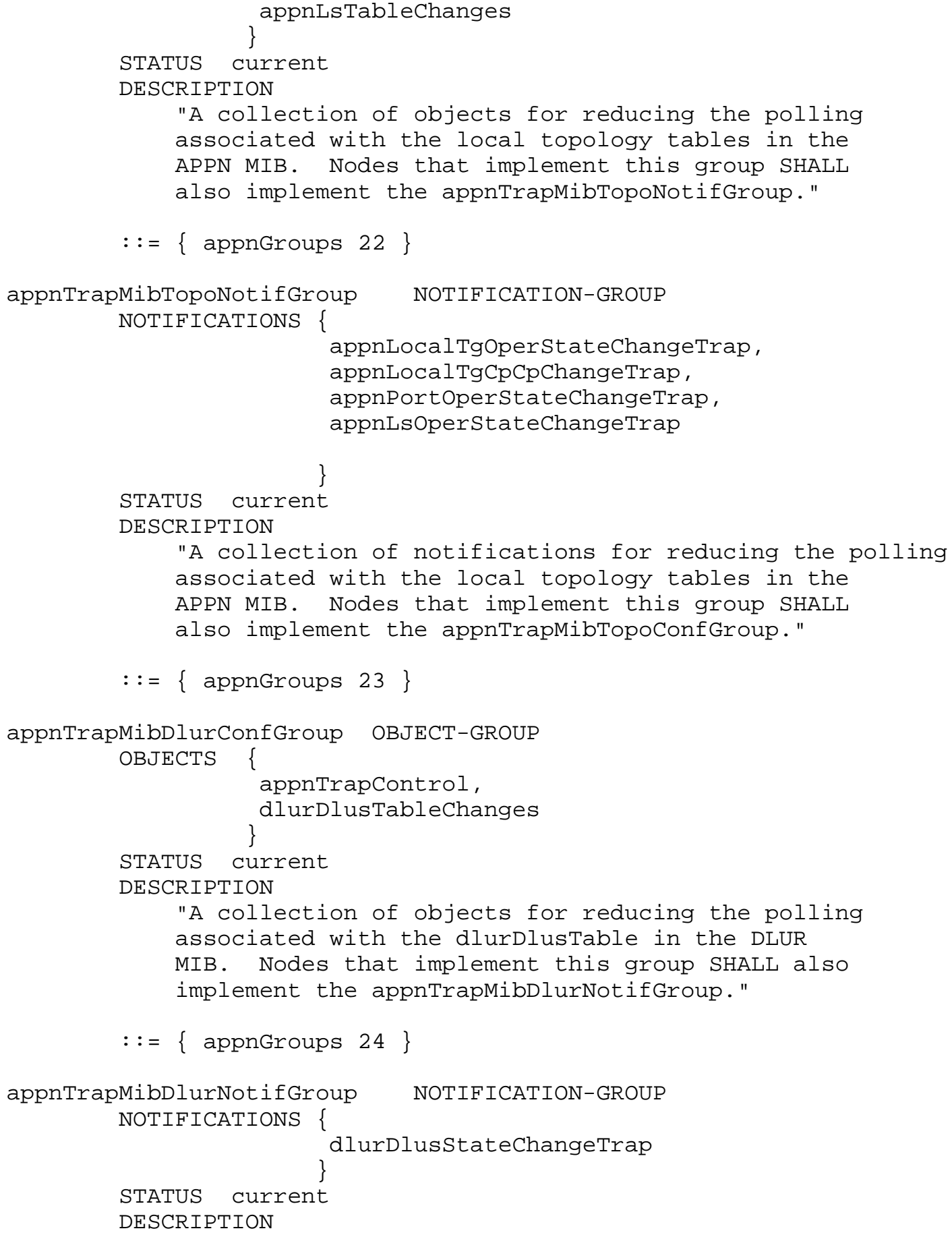




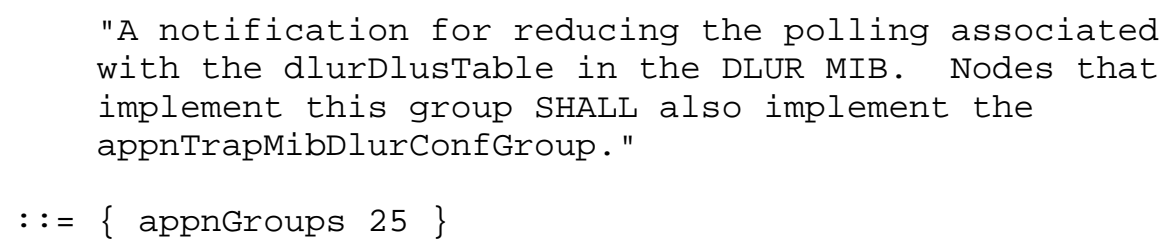

END

5. Security Considerations

Certain management information defined in this MIB may be considered sensitive in some network environments. Therefore, authentication of received SNMP requests and controlled access to management information SHOULD be employed in such environments. An authentication protocol is defined in [12]. A protocol for access control is defined in [15].

None of the read-only objects in the APPN TRAP MIB reports a password, user data, or anything else that is particularly sensitive. Some enterprises view their network configuration itself, as well as information about network usage and performance, as corporate assets; such enterprises may wish to restrict SNMP access to most of the objects in the MIB.

There is one read-write object in the APPN TRAP MIB, appnTrapcontrol. This object controls the generation of the notifications defined in the APPN TRAP MIB.

6. Intellectual Property

The IETF takes no position regarding the validity or scope of any intellectual property or other rights that might be claimed to pertain to the implementation or use of the technology described in this document or the extent to which any license under such rights might or might not be available; neither does it represent that it has made any effort to identify any such rights. Information on the IETF's procedures with respect to rights in standards-track and standards- related documentation can be found in BCP-11 [16]. Copies of claims of rights made available for publication and any assurances of licenses to be made available, or the result of an attempt made to obtain a general license or permission for the use of such proprietary rights by implementers or users of this specification can be obtained from the IETF Secretariat. 
The IETF invites any interested party to bring to its attention any copyrights, patents or patent applications, or other proprietary rights which may cover technology that may be required to practice this standard. Please address the information to the IETF Executive Director.

7. Acknowledgments

This MIB module is the product of the IETF SNA NAU MIB WG and the AIW APPN/HPR MIBS SIG.

8. References

[1] Harrington, D., Presuhn, R., and B. Wijnen, "An Architecture for Describing SNMP Management Frameworks", RFC 2271, Cabletron Systems, Inc., BMC Software, Inc., IBM T. J. Watson Research, January 1998 .

[2] Rose, M., and K. McCloghrie, "Structure and Identification of Management Information for TCP/IP-based Internets", STD 16, RFC 1155, May 1990 .

[3] Rose, M., and K. McCloghrie, "Concise MIB Definitions", STD 16, RFC 1212, March 1991.

[4] Rose, M., "A Convention for Defining Traps for use with the SNMP", RFC 1215, March 1991.

[5] Case, J., McCloghrie, K., Rose, M., and S. Waldbusser, "Structure of Management Information for Version 2 of the Simple Network Management Protocol (SNMPv2)", RFC 1902, January 1996.

[6] Case, J., McCloghrie, K., Rose, M., and S. Waldbusser, "Textual Conventions for Version 2 of the Simple Network Management Protocol (SNMPv2)", RFC 1903, January 1996.

[7] Case, J., McCloghrie, K., Rose, M., and S. Waldbusser, "Conformance Statements for Version 2 of the Simple Network Management Protocol (SNMPv2)", RFC 1904, January 1996.

[8] Case, J., Fedor, M., Schoffstall, M., and J. Davin, "Simple Network Management Protocol", STD 15, RFC 1157, May 1990.

[9] Case, J., McCloghrie, K., Rose, M., and S. Waldbusser, "Introduction to Community-based SNMPv2", RFC 1901, January 1996 . 
[10] Case, J., McCloghrie, K., Rose, M., and S. Waldbusser, "Transport Mappings for Version 2 of the Simple Network Management Protocol (SNMPv2)", RFC 1906, January 1996.

[11] Case, J., Harrington D., Presuhn R., and B. Wijnen, "Message Processing and Dispatching for the Simple Network Management Protocol (SNMP)", RFC 2272, January 1998.

[12] Blumenthal, U., and B. Wijnen, "User-based Security Model (USM) for version 3 of the Simple Network Management Protocol (SNMPV3)", RFC 2274, January 1998.

[13] Case, J., McCloghrie, K., Rose, M., and S. Waldbusser, "Protocol Operations for Version 2 of the Simple Network Management Protocol (SNMPv2)", RFC 1905, January 1996.

[14] Levi, D., Meyer, P., and B. Stewart, "SNMPv3 Applications", RFC 2273, January 1998 .

[15] Wijnen, B., Presuhn, R., and K. McCloghrie, "View-based Access Control Model (VACM) for the Simple Network Management Protocol (SNMP) ", RFC 2275, January 1998

[16] Hovey, R., and S. Bradner, "The Organizations Involved in the IETF Standards Process", BCP 11, RFC 2028, October 1996.

[17] Bradner, S., "Key words for use in RFCs to Indicate Requirement Levels", BCP 14, RFC 2119, March 1997.

[18] Clouston, B., and B. Moore, "Definition of Managed Objects for APPN", RFC 2455, November 1998.

[19] Clouston, B., and B. Moore, "Definitions of Managed Objects for DLUR", RFC 2232, November 1997. 
9. Authors' Addresses

Bob Clouston

Cisco Systems

7025 Kit Creek Road

P.O. Box 14987

Research Triangle Park, NC 27709, USA

Phone: +1 9194722333

EMail: clouston@cisco.com

Robert Moore

Dept. BRQA/Bldg. 501/G114

IBM Corporation

P.O.Box 12195

3039 Cornwallis

Research Triangle Park, NC 27709, USA

Phone: +1 9192544436

EMail: remooredus.ibm.com 
10. Full Copyright statement

Copyright (C) The Internet Society (1998). All Rights Reserved.

This document and translations of it may be copied and furnished to others, and derivative works that comment on or otherwise explain it or assist in its implementation may be prepared, copied, published and distributed, in whole or in part, without restriction of any kind, provided that the above copyright notice and this paragraph are included on all such copies and derivative works. However, this document itself may not be modified in any way, such as by removing the copyright notice or references to the Internet society or other Internet organizations, except as needed for the purpose of developing Internet standards in which case the procedures for copyrights defined in the Internet Standards process must be followed, or as required to translate it into languages other than English.

The limited permissions granted above are perpetual and will not be revoked by the Internet society or its successors or assigns.

This document and the information contained herein is provided on an "AS IS" basis and THE INTERNET SOCIETY AND THE INTERNET ENGINEERING TASK FORCE DISCLAIMS ALL WARRANTIES, EXPRESS OR IMPLIED, INCLUDING BUT NOT LIMITED TO ANY WARRANTY THAT THE USE OF THE INFORMATION HEREIN WILL NOT INFRINGE ANY RIGHTS OR ANY IMPLIED WARRANTIES OF MERCHANTABILITY OR FITNESS FOR A PARTICULAR PURPOSE. 